\title{
A single origin of the photosynthetic organelle in different Paulinella
} lineages

\author{
Hwan Su Yoon*†1, Takuro Nakayama ${ }^{\dagger 2}$, Adrian Reyes-Prieto ${ }^{\dagger 3}$, \\ Robert A Andersen ${ }^{1}$, Sung Min Boo ${ }^{4}$, Ken-ichiro Ishida ${ }^{2}$ and \\ Debashish Bhattacharya ${ }^{3}$
}

\begin{abstract}
Address: ${ }^{1}$ Bigelow Laboratory for Ocean Sciences, West Boothbay Harbor, Maine, USA, ${ }^{2}$ Graduate School of Life and Environmental Sciences, University of Tsukuba, Tsukuba, Ibaraki, Japan, ${ }^{3}$ Department of Biology and Roy J. Carver Center for Comparative Genomics, University of Iowa, Iowa City, Iowa, USA and ${ }^{4}$ Department of Biology, Chungnam National University, Daejeon, Korea

Email: Hwan Su Yoon* - hsyoon@bigelow.org; Takuro Nakayama - s0730456@ipe.tsukuba.ac.jp; Adrian Reyes-Prieto - adrianreyes@uiowa.edu; Robert A Andersen - randersen@bigelow.org; Sung Min Boo - smboo@cnu.ac.kr; Ken-

ichiro Ishida - ishida@sakura.cc.tsukuba.ac.jp; Debashish Bhattacharya - debashi-bhattacharya@uiowa.edu

* Corresponding author †Equal contributors
\end{abstract}

Published: 13 May 2009

BMC Evolutionary Biology 2009, 9:98 doi:10.1186/147|-2/48-9-98

This article is available from: http://www.biomedcentral.com/I47I-2/48/9/98

(c) 2009 Yoon et al; licensee BioMed Central Ltd.

This is an Open Access article distributed under the terms of the Creative Commons Attribution License (http://creativecommons.org/licenses/by/2.0), which permits unrestricted use, distribution, and reproduction in any medium, provided the original work is properly cited.

\begin{abstract}
Background: Gaining the ability to photosynthesize was a key event in eukaryotic evolution because algae and plants form the base of the food chain on our planet. The eukaryotic machines of photosynthesis are plastids (e.g., chloroplast in plants) that evolved from cyanobacteria through primary endosymbiosis. Our knowledge of plastid evolution, however, remains limited because the primary endosymbiosis occurred more than a billion years ago. In this context, the thecate "green amoeba" Paulinella chromatophora is remarkable because it very recently (i.e., minimum age of $\approx 60$ million years ago) acquired a photosynthetic organelle (termed a "chromatophore"; i.e., plastid) via an independent primary endosymbiosis involving a Prochlorococcus or Synechococcus-like cyanobacterium. All data regarding P. chromatophora stem from a single isolate from Germany (strain M0880/a). Here we brought into culture a novel photosynthetic Paulinella strain (FKOI) and generated molecular sequence data from these cells and from four different cell samples, all isolated from freshwater habitats in Japan. Our study had two aims. The first was to compare and contrast cell ultrastructure of the M0880/a and FKOI strains using scanning electron microscopy. The second was to assess the phylogenetic diversity of photosynthetic Paulinella to test the hypothesis they share a vertically inherited plastid that originated in their common ancestor.
\end{abstract}

Results: Comparative morphological analyses show that Paulinella FKOI cells are smaller than M0880/a and differ with respect to the number of scales per column. There are more distinctive, multiple fine pores on the external surface of FKOI than in M0880/a. Molecular phylogenetic analyses using multiple gene markers demonstrate these strains are genetically distinct and likely comprise separate species. The well-supported monophyly of the Paulinella chromatophora strains analyzed here using plastid-encoded I6S rRNA suggests strongly that they all share a common photosynthetic ancestor. The strain M0880/a is most closely related to Japanese isolates (KanazawaI, -2, and Kaga), whereas FKOI groups closely with a Kawaguchi isolate.

Conclusion: Our results indicate that Paulinella chromatophora comprises at least two distinct evolutionary lineages and likely encompasses a broader taxonomic diversity than previously thought. The finding of a single plastid origin for both lineages shows these taxa to be valuable models for studying post-endosymbiotic cell and genome evolution. 


\section{Background}

The origin of the first photosynthetic organelle (plastid) is explained by primary endosymbiosis, whereby a nonphotosynthetic protist engulfed and retained a cyanobacterium as a cytoplasmic organelle $[1,2]$. A variety of plastid-derived and some nuclear sequence data, as well as plastid-associated traits such as the composition and evolutionary history of the protein translocons [3], and solute transporters embedded in the inner organelle membrane suggest that descendants of this 'primary' photosynthetic eukaryote were the ancestors of the putative supergroup Plantae. The Plantae is comprised of the red, green (including land plants), and glaucophyte algae [4,5]. Via eukaryote-eukaryote (secondary and tertiary) endosymbiosis, photosynthesis and its associated genes spread thereafter into other eukaryotic groups (e.g., euglenoids, diatoms, and dinoflagellates; [6-10]). Despite its importance to eukaryote evolution, our understanding of primary plastid origin remains limited because the endosymbiosis occurred $>1$ billion years ago $[11,12]$. This dilemma has, however, a potential solution given the recently clarified evolutionary history of Paulinella chromatophora. This little-known testate, filose amoeba [13], which is a cercomonad species (supergroup Rhizaria), contains two blue-green photosynthetic inclusions termed chromatophores [14]. There are no known fossils of Paulinella, however, euglyphid-like testate amoebae have a long fossil history, occurring in sediments dated from $742-770$ million years ago $[15,16]$. Importantly, $P$. chromatophora is the only known case of an independent primary photosynthetic organelle acquisition (from a prey cell related to extant Prochlorococcus/Synechococcuslike cyanobacteria), putatively recapitulating the process that gave rise to the Plantae plastid [17-20]. This makes $P$. chromatophora an outstanding model for elucidating plastid acquisition and post-endosymbiotic genome evolution. Two key reasons why it is believed the chromatophores (= plastids) of $P$. chromatophora are bona fide organelles rather than temporary photosynthetic inclusions (for details, see [21]) are the apparent regulation of plastid division by the amoeba and the loss of $2 / 3$ of plastid coding potential through outright gene loss or transfer to the nucleus (i.e., from ca. $3 \mathrm{Mb}$ in free-living Prochlorococcus/Synechococcus cyanobacteria to $1.02 \mathrm{Mb}$ in the $P$. chromatophora plastid, [22]). Paulinella plastid genome size is, however, far greater than the ca. 100-200 $\mathrm{Kb}$ for typical plastid genomes from algae and plants suggesting it is likely a "work in progress" (for details, see [23]).

Since its discovery by Lauterborn [14], four different Paulinella species have been reported - the photoautotrophic $P$. chromatophora, which contains two plastids, is clearly separated from its three heterotrophic sister species that lack a plastid (i.e., P. ovalis, $P$. intermedia, and $P$. indentata), although all share a typical oval-shaped cell morphology that consists of five rows of silicate scales (see Table 1, [24-26]). The phylogenetic relationship between these four Paulinella species is unknown because of a lack of sequence data from heterotrophic Paulinella species. However, it is obvious that $P$. chromatophora (as a representative of the genus Paulinella) and Euglypha are closely related based on nuclear SSU rDNA trees $[13,27]$. The derived position of $P$. chromatophora among the Paulinellidae and the known ability of $P$. ovalis to ingest cyanobacteria [25] make it a reasonable assumption that the primary plastid endosymbiosis occurred in $P$. chromatophora after its split from heterotrophic ancestors.

The minimum age of the endosymbiosis is postulated to be ca. $60 \mathrm{Ma}$ based on the mode and tempo of plastid genome reduction [22]. Given these data, it is of high interest to isolate other photosynthetic Paulinella strains/ species to facilitate in-depth study of post-endosymbiotic

Table I: Comparison of morphological characters among Paulinella species.

\begin{tabular}{|c|c|c|c|c|c|c|}
\hline & $\begin{array}{l}\text { This study } \\
\text { FKOI } \\
(\mathrm{n}=17)\end{array}$ & $\begin{array}{l}\text { P. chromatophora } \\
\text { M0880/a } \\
(\mathrm{n}=13)\end{array}$ & $\begin{array}{l}\text { Lauterborn } 1895, \\
\text { Kies } 1974\end{array}$ & $\begin{array}{l}\text { P. ovalis } \\
\text { Johnson et al } 1988\end{array}$ & $\begin{array}{l}\text { P. intermedia } \\
\text { Vørs } 1993\end{array}$ & $\begin{array}{l}\text { P. indentata } \\
\text { Hannah et al } 1996\end{array}$ \\
\hline Plastid & + & + & + & - & - & - \\
\hline Length $(\mu \mathrm{m})$ & $15-17$ & $23-27$ & $20-30$ & 4.5 & 6.6 & $11-17$ \\
\hline Width $(\mu \mathrm{m})$ & $10-11$ & $16-20$ & $15-20$ & 3 & 2.9 & $8-10$ \\
\hline $\begin{array}{l}\text { No. of scales/ } \\
\text { column }\end{array}$ & $10-11$ & $12-14$ & $11-12$ & $5-6$ & $6-7$ & $6-7$ \\
\hline No. of columns & 5 & 5 & 5 & 5 & NA & Staggered rows \\
\hline Oral scales & 5 & 3 & 3 & 3 & 3 & $2-4$ \\
\hline $\begin{array}{l}\text { Fine-pored } \\
\text { external scales }\end{array}$ & $+($ distinct $)$ & $+($ not distinct $)$ & NA & + & - & $\begin{array}{l}+(3-4 \text { rows of } \\
\text { pores at the end of } \\
\text { scales })\end{array}$ \\
\hline Scale feature & $\begin{array}{l}\text { "Sieve-plate" on the } \\
\text { internal surface }\end{array}$ & $\begin{array}{l}\text { "Sieve-plate" on the } \\
\text { internal surface }\end{array}$ & $\begin{array}{l}\text { "Sieve-plate" on the } \\
\text { internal surface }\end{array}$ & $\begin{array}{l}\text { One-ridges and } \\
\text { hollow scales }\end{array}$ & Smooth scales & $\begin{array}{l}\text { Two-ridges and } \\
\text { hollow scales }\end{array}$ \\
\hline
\end{tabular}


genome evolution in distinct lineages that potentially share a common ancestral endosymbiont. Paulinella chromatophora has been reported from around the world, including sites in Switzerland [28], the United Kingdom [29], and the United States [30,31], however these were simple statements of occurrence without the deposition of voucher samples. This depauperate history of collection apparently reflects the rarity of $P$. chromatophora in nature and difficulties in its culture. Therefore, all morphological and ultrastructural studies stem from samples collected in Germany [19,32]. Recent molecular phylogenetic and genomic studies also relied on strain M0880/a that was isolated in Germany $[13,17,22,33,34]$. Here, we isolated photosynthetic Paulinella cells from several freshwater sites in Japan and established a new strain in culture (FK01). We then conducted comparative morphological and molecular phylogenetic analysis of these taxa, focus- ing on the Japanese FK01 and the German M0880/a strains.

\section{Results and discussion \\ Morphology and ultrastructure of Paulinella}

Morphological comparisons between Paulinella FK01 and M0880/a strains were done using scanning electron microscopy (SEM). The general morphological characters we observed for M0880/a (Fig. 1D, E) are similar to the original description [14] and a previous study [19]. The test is ovoid $(23-27 \times 16-20 \mu \mathrm{m} ; \mathrm{n}=13)$ and covered with silica scale plates. The anterior end has a narrow aperture $(3-5.6 \mu \mathrm{m})$ that is comprised of three oral scales (see asterisks in Fig. 1D) extending from the cell body as a "neck" (see N in Fig. 1D). Within this neck, two slightly curved oral scales abut each other between a membranous operculum with the third scale covering the edge of the two oral scales (Fig. 1E). Below the oral scales, five

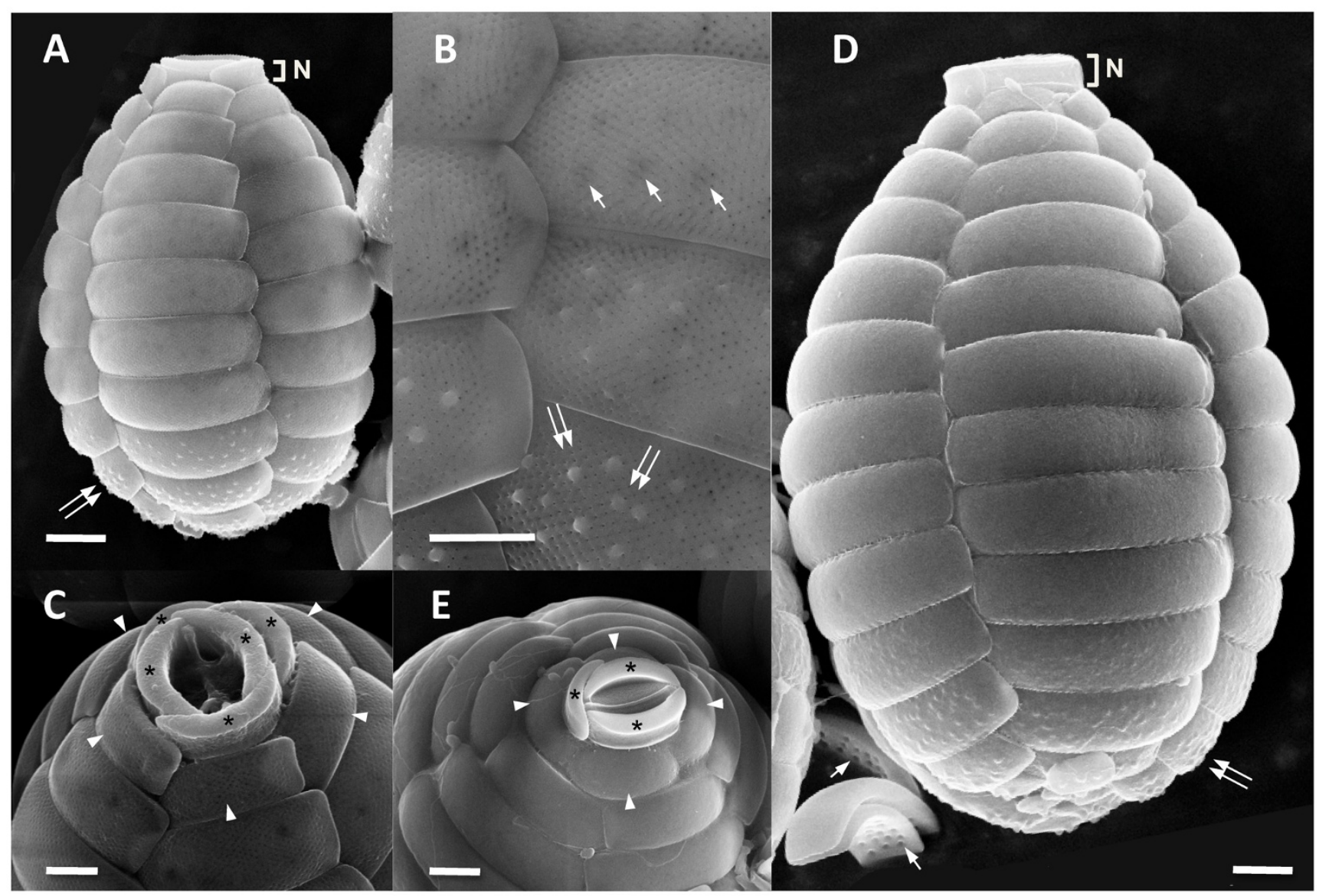

Figure I

SEM images of photosynthetic Paulinella strains FKOI (A - C) and M0880/a (D, E). FKOI is smaller in cell size than M0880/a (i.e., the scale bar is the same in $A$ and D). Distinctive, multiple fine pores are present on the surface of scales of FKO I (B). The five (C) oral scales (asterisks) are shown from FKOI, whereas only three (E) are found in M0880/a. The projecting oral scales $(N)$, first row of body scales (arrow head), "sieve-plate" in internal surface (arrow), and pustules (double arrow) are indicated. Scale bars (A, D, E = $2 \mu \mathrm{m} ; \mathrm{B}, \mathrm{C}=\mathrm{I} \mu \mathrm{m}$ ). 
descending columns of scales cover the test. A total of 12 - 14 scales are found in each column with the first row containing four scales instead of five (see arrow head in Fig. 1D). The scales around the anterior and posterior cell regions are smaller than in the middle. Three or four rows of scales from the posterior end have dozens of pustules on the surface of each scale. The external surface of the rest of the body scales is smooth. The internal surface of the scales that are shown in Fig. 1D (see arrows in inverted scales) have $12-18$ pores per scale which were reported as "sieve-plates" in a previous study [19].

Cells of strain FK01 are clearly distinguished from the original description of $P$. chromatophora (i.e., M0880/a). These Paulinella cells are of a relatively smaller size (15 $17 \times 10-11 \mu \mathrm{m} ; \mathrm{n}=17$ ) than in M0880/a (see, Fig. 1AC) and there are between $10-11$ scales per column. There are more distinctive, multiple fine pores on the external surface of scales in FK01 than in M0880/a (see, Fig. 1B). The fine-pored external surface was reported in newly formed scales in the heterotrophic species $P$. ovalis [25]. Another heterotrophic species, $P$. indentata has a single row of fine-pores along the scale but three or four rows on the end of the scales [24]. There are five oral scales in the aperture $(2.4-3.6 \mu \mathrm{m})$, which is comprised of two main scales between a membranous operculum and three thinner scales that cover the main oral scales (see asterisks in Fig. 1C). The oral scales of FK01 are not projected outward to the same extent as in M0880/a (Fig. 1A vs. 1D, indicated with $\mathrm{N}$ ), and the first row of body scales consists of five not four scales (see arrow head in Fig. 1A, C). Pustules on the four rows of posterior scales (see double arrow in Figs. 1A-B) are more distinct than those in the M0880/a strain. The size of pustule-covered scales gradually increases from the centre to the outside in a counter-clockwise direction. Around 10 - 20 internal "sieve-plate" pores are also found in FKO1 and are detectable in SEM images taken from outside the cell, particularly in the posterior region (see arrows in Fig. 1B).

Table 1 shows a comparison of key morphological characters from different Paulinella species. Traits such as cell size, number of scales per column, and number of oral scales have been used to define species. For example, $P$. intermedia is similar to $P$. ovalis in size and the number of scales per column but it differs from the latter species by possessing flat scales and a wider oral aperture [26]. Due to the presence of two plastids in the cytosol, the FK01 and M0880/a strains are clearly distinguished from other Paulinella species. In turn, FK01 is distinct from M0880/a with regard to cell size, number of scales, number of oral scales, and by having distinct fine-pores in the body scales.

\section{Molecular phylogenetic analysis}

Given the obvious morphological differences described above, we used gene sequences to test the evolutionary relationship between the two strains. Multiple markers were used for this purpose and we first present maximum likelihood (ML) trees inferred from nuclear $18 \mathrm{~S}$ rDNA and a concatenated data set of plastid 16S + 23S rDNA (Figs. 2A, B). Given that filose amoebae such as P. chromatophora and Euglypha are consistently recovered as members of the Rhizaria (e.g., [13,27]), we chose to include only $18 \mathrm{~S}$ rDNA sequences from members of this putative supergroup [35]. The $18 \mathrm{~S}$ rDNA tree shows a monophyletic grouping of the two Paulinella strains, suggesting they share a common photosynthetic 'host' ancestor. This hypothesis is substantiated by the plastid rDNA tree (Fig. 2B), that shows a monophyletic grouping of M0880/a and FK01 as sister to Synechococcus- and Prochlorococcus-type cyanobacteria as previously described for M0880/a [22,34]. The $18 \mathrm{~S}$ rDNA tree provides high bootstrap and Bayesian support (posterior probability $>0.95$ for all thick nodes in the trees; $100 \%$ bootstrap support in both RAxML [RML] and PhyML [PML] analyses) for a clade that unites both $P$. chromatophora strains with a group of uncultured marine environmental samples (i.e., GenBank accession numbers; AB275059, EF526891, see [36]). Because there are no sequence data available from heterotrophic Paulinella species with a taxonomic identification, we could not provisionally identify the source of the environmental samples. However, given that all described photosynthetic $P$. chromatophora are derived from freshwater environments $[14,19]$ and the present work], these environmental sequences likely represent a marine sister group within the Paulinellidae (e.g., P. ovalis, $P$. indentata $[24,25])$. The Paulinellidae is closely related ( $90 \%$ RML, PML) to other euglyphids such as Tracheoeuglypha, Cyphoderia, and Euglypha species.

Trees inferred from actin and $f t s Z$ protein sequences are shown in Figures 3 and 4, respectively. The actin tree provides both bootstrap and Bayesian support for the existence of a branch that unites M0880/a and FK01 (89\% RML, 95\% PML), which in turn is sister to other euglyphid taxa (57\% RML, absence of Bayesian support) within the Rhizaria. The overall topology for Rhizaria actins (Fig. 3) is consistent with previous analyses [37]. This monophyletic clade, although surrounded by many internal nodes that are only weakly supported, shows unequivocal sequence divergence between M0880/a and FK01. Pairwise analysis of synonymous (Ks) and non-synonymous (Ka) substitution rates between the Paulinella actin coding regions are 1.0023 and 0.0181 , respectively $(\mathrm{Ka} / \mathrm{Ks}=$ 0.0181 ). This ratio is comparable to actin sequence differences between two green algal Ostreococcus species (i.e., $O$. tauri vs. O. lucimarinus; $\mathrm{Ks}=1.2489, \mathrm{Ka}=0.0085, \mathrm{Ka} / \mathrm{Ks}=$ 0.0068 ), two multicelluar liverworts (Pellia endiviifolia vs. 

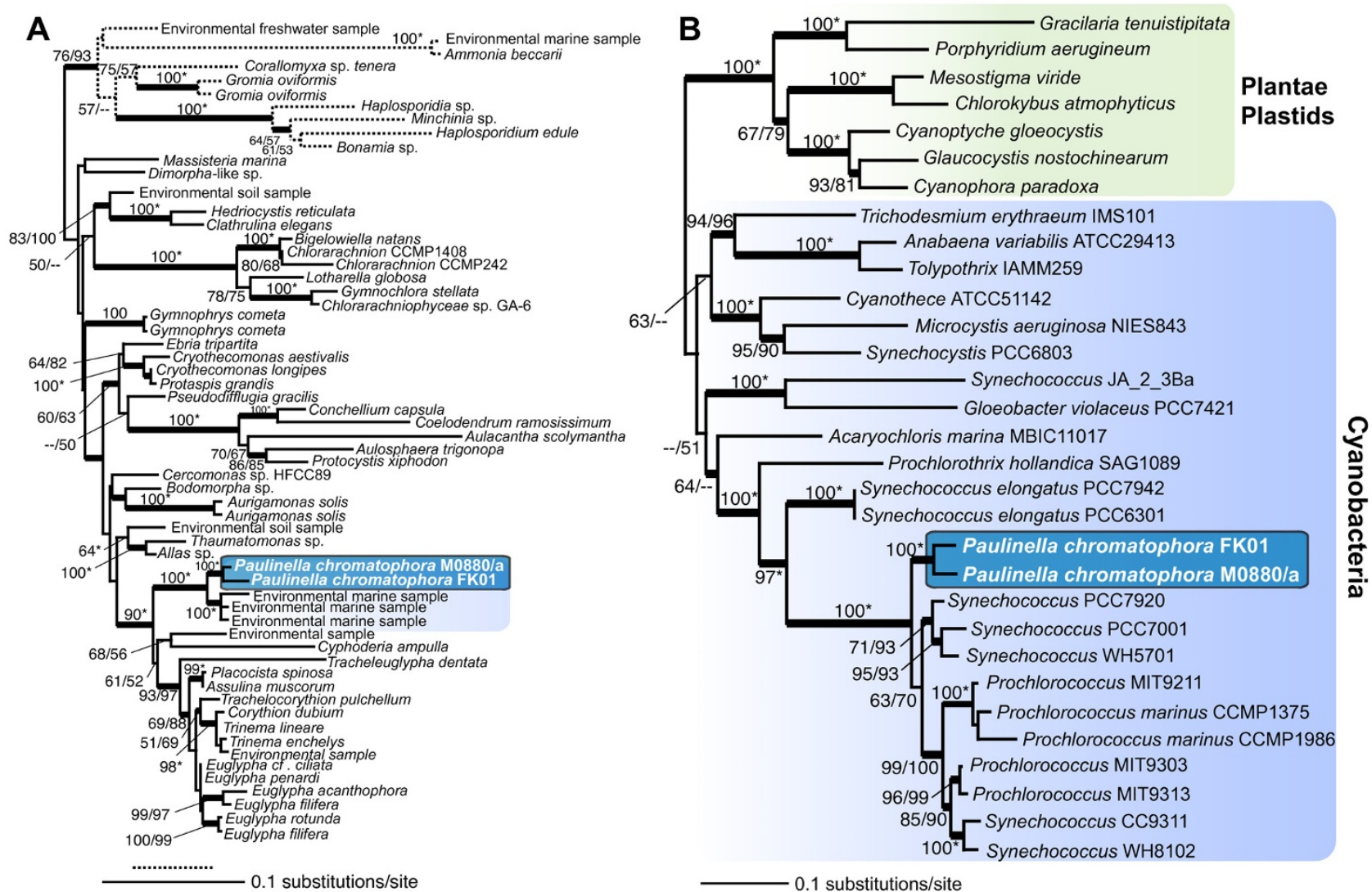

\section{Figure 2}

(A) RAxML phylogenetic tree of nuclear I8S rDNA from Rhizaria with the root placed on the branch leading to the Foraminifera. (B) RAxML phylogenetic tree of a concatenated data set of plastid-encoded I6S + 23S rDNA with the root placed on the branch leading to Plantae plastids. The numbers at the nodes of both trees show support values derived from a RAxML bootstrap analysis followed by those from a PhyML analysis. When both values are the same, then this is marked with an asterisk and when a node is not resolved with a method than this is denoted with dashes. Only bootstrap values $\geq 50 \%$ are shown. The thick branches have a Bayesian posterior probability $>0.95$. Branch lengths are proportional to the number of substitutions per site (see scale bar).

P. borealis; $\mathrm{Ks}=0.8396, \mathrm{Ka}=0.0037, \mathrm{Ka} / \mathrm{Ks}=0.0044)$, and different genera of yeasts (Saccharomyces vs. Kluyveromyces, $\mathrm{Ks}=0.4541, \mathrm{Ka}=0.0178, \mathrm{Ka} / \mathrm{Ks}=0.0300 ;$ Saccharomyces cerevisiae vs. Pichia stipitis, $\mathrm{Ks}=0.7798, \mathrm{Ka}=0.0221, \mathrm{Ka} / \mathrm{Ks}$ $=0.0283$ ). These results suggest that M0880/a and FK01 are significantly diverged from each other and likely constitute distinct species. This hypothesis is consistent with the plastid-derived gene data for rDNA (Fig. 2B) and ftsZ (Fig. 4). Interestingly, the estimated Ks value between fts $Z$ sequences from M0880/a and FK01 exceed the expected confidence limits (i.e., >> 1.0) likely indicating these plastid-encoded genes are undergoing a high nucleotide substitution rate possibly as a result of the genome reduction process. Taken together, our results indicate that after the single acquisition of the photosynthetic organelle, the $P$. chromatophora ancestor gave rise to at least two distinct lineages.

To advance our understanding of photosynthetic Paulinella, we generated plastid-encoded 16S rDNA sequences from environmental samples from four different sites in Japan. We amplified the gene directly using a small number of cells that were manually isolated from materials collected at each site (see Methods and Materials). The expanded rDNA tree of photosynthetic Paulinella (Fig. 5) shows that all of the isolates cluster together with robust bootstrap and Bayesian support (99\% RML, 98\% PML) with a sister-group relationship to Synechococcus/ Prochlorococcus, confirming the single origin of the plastid in these Paulinella. The phylogeny also indicates that the 


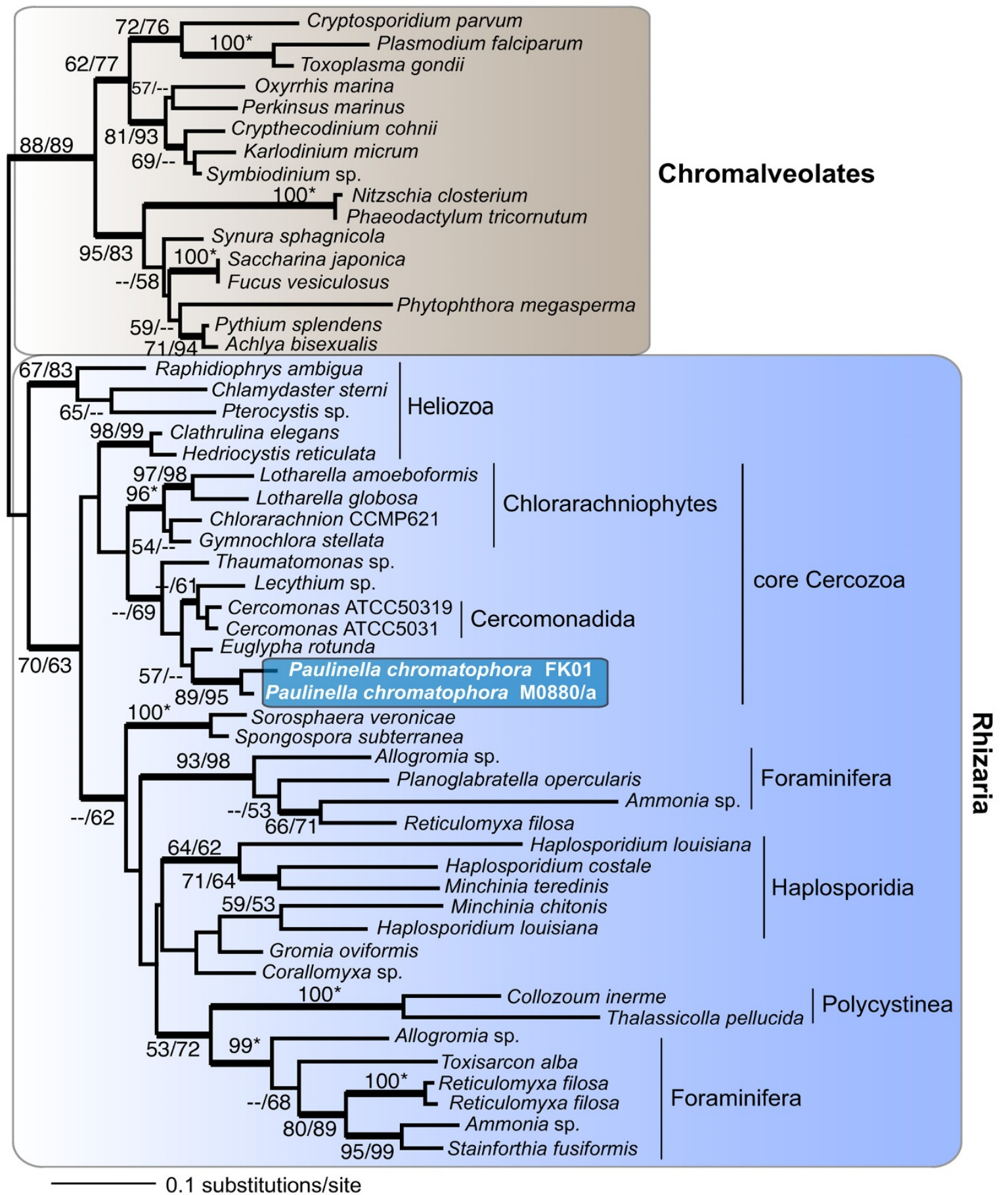

Figure 3

RAxML phylogenetic tree of actin sequences from photosynthetic Paulinella spp. with the root placed on the branch leading to chromalveolate taxa (i.e., stramenopiles + alveolates). The numbers at the nodes show support values derived from a RAxML bootstrap analysis followed by those from a PhyML analysis. When both values are the same, then this is marked with an asterisk and when a node is not resolved with a method than this is denoted with dashes. Only bootstrap values $\geq 50 \%$ are shown. The thick branches have a Bayesian posterior probability $>0.95$. Branch lengths are proportional to the number of substitutions per site (see scale bar). 


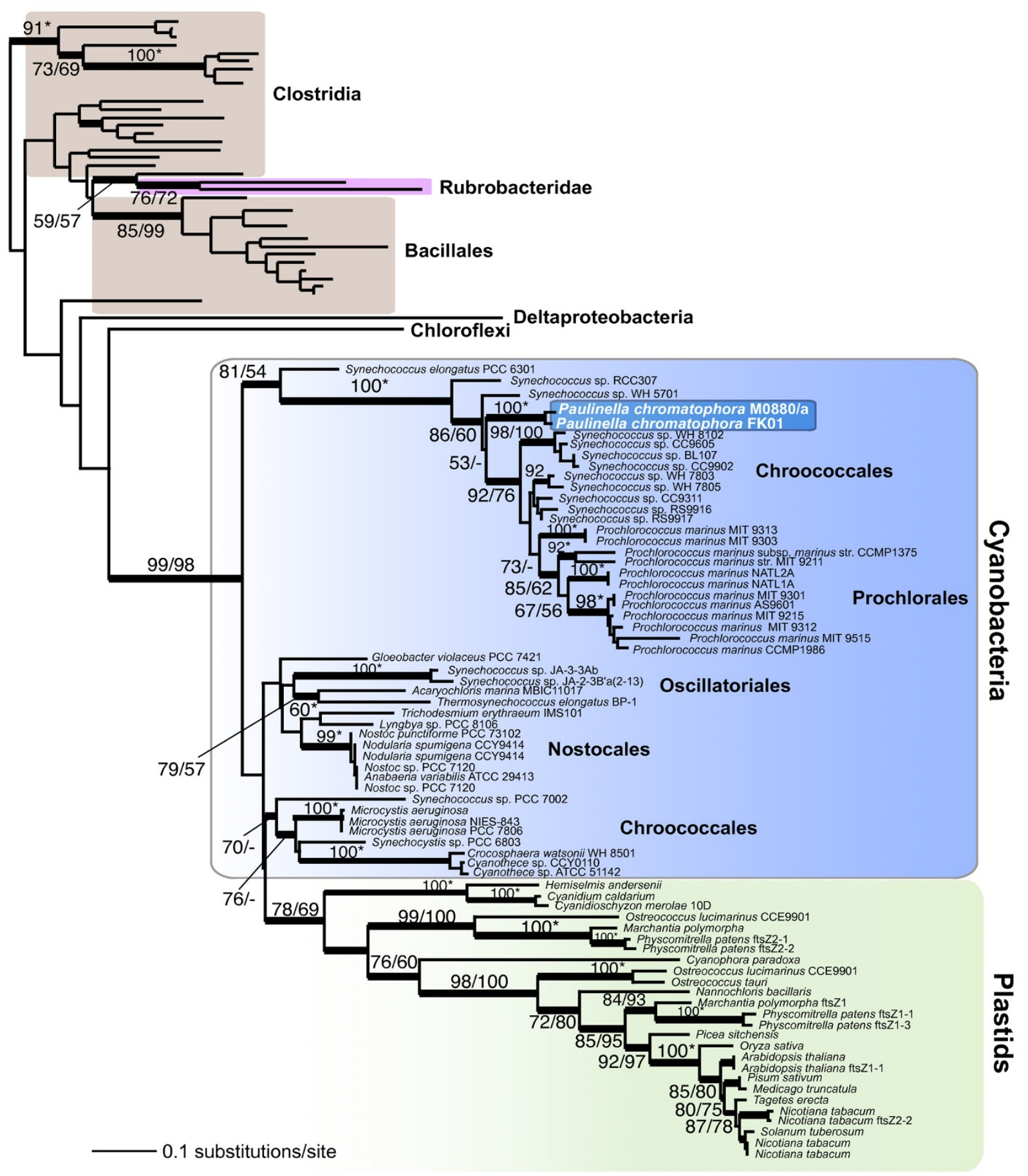

\section{Figure 4}

RAxML phylogenetic tree of plastid-encoded ftsZ from photosynthetic Paulinella spp. with the root placed on the branch leading to Clostridia. The numbers at the nodes show support values derived from a RAxML bootstrap analysis followed by those from a PhyML analysis. When both values are the same, then this is marked with an asterisk and when a node is not resolved with a method than this is denoted with dashes. Only bootstrap values $\geq 50 \%$ are shown. The thick branches have a Bayesian posterior probability $>0.95$. Branch lengths are proportional to the number of substitutions per site (see scale bar). 


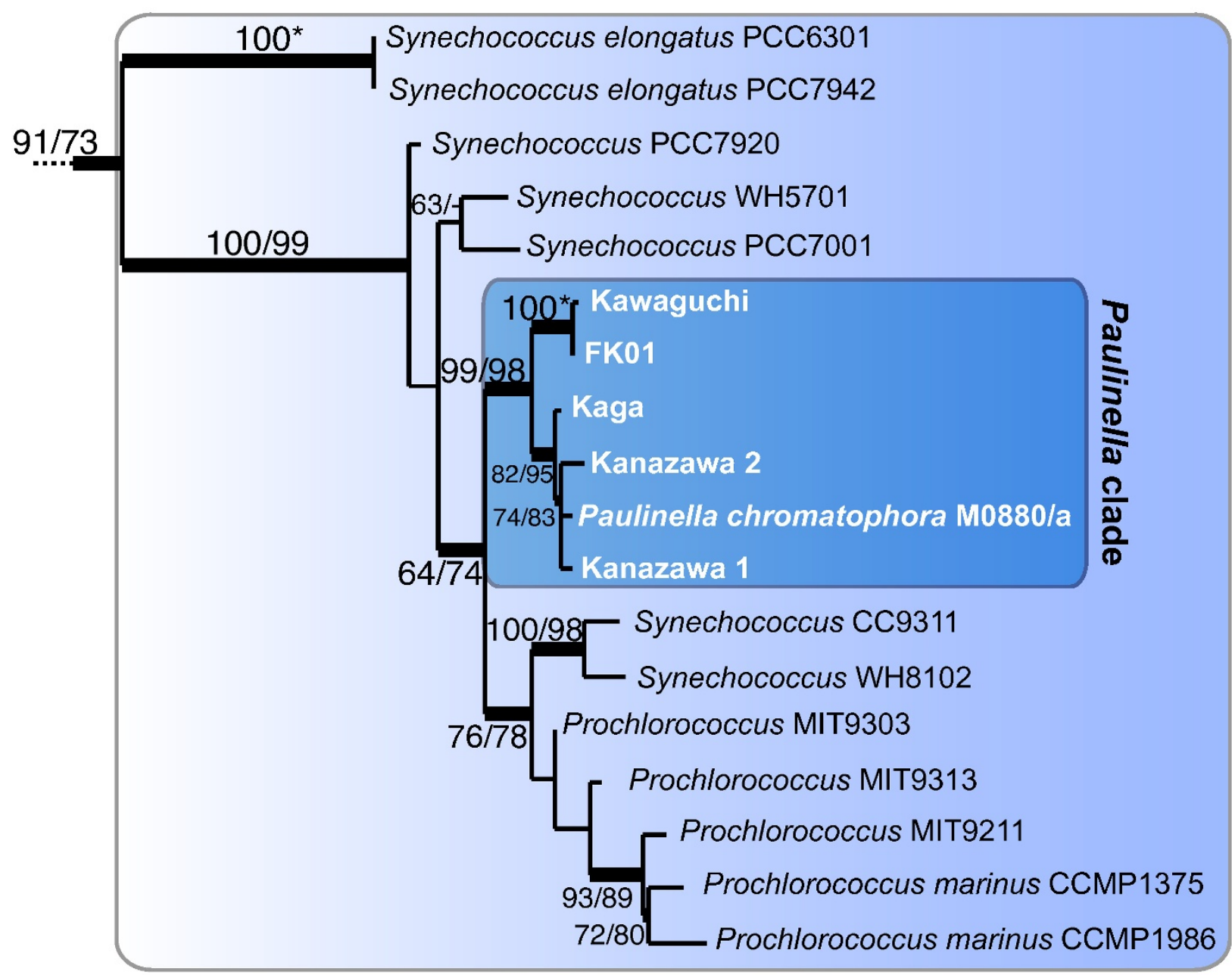

0.05 substitutions/site

\section{Figure 5}

RAxML phylogenetic tree of plastid I6S rDNA from photosynthetic Paulinella spp. with the larger tree (available upon request from HSY) removed at the branch leading to cyanobacteria. The numbers at the nodes show support values derived from a RAxML bootstrap analysis followed by those from a PhyML analysis. When both values are the same, then this is marked with an asterisk and when a node is not resolved with a method than this is denoted with dashes. Only bootstrap values $\geq 50 \%$ are shown. The thick branches have a Bayesian posterior probability $>0.95$. Branch lengths are proportional to the number of substitutions per site (see scale bar).

Paulinella isolates are split into two distinct clades. One clade includes the M0880/a strain that is closely related to isolates from Kaga and Kanazawa-1 and -2, whereas the second includes the newly isolated FK01strain and a field isolate from Lake Kawaguchi (HSY et al. unpublished data). This topology is surprising because it does not provide evidence for geographic separation (i.e., Germany vs. Japan), but rather shows the German strain M0880/a might share a most recent common ancestor with Japanese isolates (i.e., from Kanazawa and Kaga). It is possi- ble, however, that photosynthetic Paulinella species are globally distributed and we simply lack data from other sites to demonstrate this result. In any case, our results suggest these fascinating organisms are unlikely to be a relict branch of filose amoebal evolution but may be broadly distributed with many more living taxa than previously thought. Assessing further the biodiversity of this group and providing a taxonomic description of species are key next steps in understanding the biology of Paulinella (HSY et al., work underway). 


\section{Conclusion}

Our results provide sufficient data to address the major aims of this study. The morphological and molecular data show that M0880/a and the newly isolated FK01 are distinct species (see Table 1), whereas phylogenetic analyses of these strains and other cells collected in nature demonstrate that all of the photosynthetic Paulinella share a plastid derived from a single primary endosymbiosis. This is an important finding because the genus Paulinella provides a set of diverged lineages that can be used to study the process of post-endosymbiotic plastid evolution using genomic and proteomic methods. One keystone process in this regard is endosymbiotic gene transfer (EGT), driven by a gene transfer ratchet (e.g., [38,39]) that relocates endosymbiont genes to the host nucleus. This is followed in some cases by endosymbiont gene activation and import of the encoded proteins into the plastid. How this occurs in related lineages and which genes are the primary targets for EGT can now be explored in detail by studying the nuclear genome of different photosynthetic Paulinella. Nowack and colleagues [22] reported that many cyanobacterial transporters were lost in the endosymbiont genome but the question remains whether they vanished or have been transferred to the nucleus for cooption in other host functions or retargeting to the organelle. A recent study by Nakayama and Ishida [40] described for the first time a nuclear encoded photosynthetic gene in FK01 of cyanobacterial origin. The cDNA encoding psaE contains a putative polyadenylation signal (AGTAAA) and a poly(A) sequence at the 3 ' terminus, whereas the nuclear locus contains two putative spliceosomal introns. These data provide strong evidence for EGT of the $p s a \mathrm{E}$ gene and we expect to find many more examples of this phenomenon when the nuclear genome of Paulinella species is completed (HSY, DB work underway). In this regard, the current tree provides at least two distinct clades in which such questions about post-endosymbiotic EGT can be studied using genomic methods. Preliminary sequence data from the FK01 plastid genome, for example, show that it differs both with respect to gene content and gene order when compared to the published [22] M0880/a genome (ARP, DB, HSY unpublished data).

\section{Methods}

\section{Sampling and Establishment of the FKOI Strain}

Paulinella chromatophora strain (FK01) was collected from Daigo, Ibaraki Prefecture, Japan, and cells were isolated into culture by Takeshi Nakayama at the University of Tsukuba. The FK01 strain was then transferred to the Provasoli-Guillard National Center for Culture of Marine Phytoplankton (CCMP). Michael Melkonian from the University of Cologne, Germany kindly provided the German strain M0880/a to the CCMP. Both strains were maintained at the CCMP using DY-V medium at $20^{\circ} \mathrm{C}$ in flat plastic culture flasks with a 14/10 hr light/dark cycle.

\section{Light Microscopy}

General cell morphology, including the pseudopodia, was examined using a Zeiss Axioimager M1 light microscope.

\section{Scanning Electron Microscopy}

Cells were harvested with centrifugation, fixed (4\% osmium tetroxide, $8 \%$ glutaraldehyde in TE buffer) and dehydrated $(35,50,75,95$, and $100 \%$ EtOH $)$, mounted on stubs and carbon coated a Denton Vacuum sputtercoater/evaporator (Desk IV, with Carbon Accessory). Cells were observed in a Zeiss Supra 25 field emission SEM.

\section{DNA Amplification and Sequencing}

Total genomic DNA from Paulinella cultures was extracted using the DNeasy Plant Mini Kit (Qiagen). PCR was done with degenerate primers for actin (Ac245F: AACTGGGAYGAYATGGARAAGAT; AC1500R: AYCCACATCTGCTGRAANGTG), $18 \mathrm{~S}$ rDNA (EukA: AACCTGGTTGATCCTGCCAG; EukB: TGATCCTTCTGCAGGTTCACCTAC, [38]) FtsZ (ftsZ150F: AGYAATGCNGTSAAYCGVATGAT; ftsZ1000R: CACGTSACBGTGATYGCCACVGG), and specific primers for the Paulinella plastid genes 16S rDNA (16Snt5F: CTTAACACATGCAAGTGCAACG; 16Snt725F: CCATAACTGACGCTCATGGACG; 16Snt725F: CGTCCATGAGCGTCAGTTATGG; 16Snt1500R: GTACGGCTACCTTGTTACGAC) and 23S rDNA (23Snt27F: GATACCTTGGCACACAGAGG; 23Snt1224F: GCAGCTTCGGTAAAACGCTTAG) genes. We amplified and determined partial actin sequences from both Paulinella FK01 and M0880/a strains, and we determined partial sequences for the 18S rDNA, 16S rDNA, 23S rDNA, and $f t s Z$ genes from the FK01 strain. All reactions were done with an initial denaturation step at $94^{\circ} \mathrm{C}$ for $10 \mathrm{~min}$, followed by 35 cycles of $94^{\circ} \mathrm{C}$ for $1 \mathrm{~min}, 50^{\circ} \mathrm{C}$ for $1 \mathrm{~min}$, and $72^{\circ} \mathrm{C}$ for $2 \mathrm{~min}$, concluding with a $10 \mathrm{~min}$ extension at $72^{\circ} \mathrm{C}$. P. chromatophora plastid-encoded $16 \mathrm{~S}$ rDNA sequences were amplified using PCR from four additional isolates from different sites in Japan. The cells were collected from two ponds located in Kanazawa (Kanazawa-1 and -2), from a pond located in Kaga Ishikawa Prefecture, and from Lake Kawaguchi in Yamanashi Prefecture, Japan. Because P. chromatophora is difficult to culture in the laboratory, we amplified 16S rDNA fragments directly from isolated cells. To avoid contamination, isolated cells were washed 10 times with sterilized water before mechanical disruption of the scaly cover and the cell membrane. Cell breakage was corroborated by light microscopy. The cell homogenate was transferred directly to microfuge tubes to be used as DNA template for PCR amplification using universal primers (U16F1: AGAGTTTGATCCTGGCTCAG, U16R1: ACGGCTACCTTGTTACGACTT). PCR products were purified (QIAquick PCR Purification kit, Qiagen) and either directly sequenced (BigDye ${ }^{\mathrm{TM}}$ Terminator Cycle Sequencing Kit, PE-Applied Biosystems) or cloned (TOPO 
4 PCR vector, Invitrogen) prior to sequencing. All sequences are available in GenBank (accession numbers from FJ456915 - FJ456920 and FJ184058 - FJ184061).

\section{Phylogenetic Analysis}

Sequence sets (protein or nucleotide) were aligned with Muscle [41], and manually refined (alignments are available upon request). The ML trees were inferred with RAxML (VI-HPC, v2.2.1, [42]) using the WAG substitution model, gamma distribution ('PROTGAMMAWAG' implementation), with 4 discrete rate categories, and starting from a random tree. Branch support was evaluated with 100 bootstrap replicates using both RAxML (WAG substitution model and the 'PROTCATWAG' implementation) and PhyML [43] (WAG $+\Gamma$ substitution model, and parameters estimated during the tree search). We also did Bayesian analyses with each data set using MrBayes 3.1.1 [44] and the WAG $+I+\Gamma$ model of sequence evolution for the protein alignments and GTR I $+\Gamma$ for the rDNA data. For each alignment, Metropoliscoupled Markov chain Monte Carlo from a random starting tree and 2 runs were started simultaneously. The Bayesian analyses were run for 1,000,000 generations with trees sampled each 100 cycles. Four chains were run simultaneously of which three were heated and one was cold, with the initial 250,000 cycles (2,500 trees) being discarded as the 'burn in'. A consensus tree was made with the remaining phylogenies to determine the posterior probabilities at each node.

\section{Nucleotide Substitution Rate Calculation}

Actin sequences at both protein and nucleotide levels of Ostreococcus tauri (jgi, Ostta4 29599), Ostreococcus lucimarinus (gi, 144581780), Saccharomyces cerevisiae (gi, 42742172), Pichia stipitis (gi, 126257874), and Kluiveromyces lactis (gi, 50306650) were obtained from JGI and GenBank. Paired protein sequences were aligned with Muscle [41], thereafter, protein pairwise alignments were used as a reference to align the corresponding coding nucleotide sequences to identify the correct reading frame. The rate of synonymous (Ks) and non-synonymous (Ka) substitutions were estimated from the resulting nucleotide alignments using PAML [45].

\section{Authors' contributions}

HSY oversaw data collection and analysis, led the morphological analysis, contributed to writing; ARP led the molecular phylogenetic analysis, contributed to writing; RAA contributed to culture maintenance and SEM analysis, and contributed to writing; SMB contributed to financial support for the Paulinella gene sequencing, and contributed to writing; KI contributed to culture establishment of FK01; TN contributed to culture establishment of FK01 and generated 16S rDNA sequences from four addi- tional isolates; DB oversaw data collection and analysis, contributed to writing and revision.

\section{Acknowledgements}

The authors wish to thank Dr. Takeshi Nakayama at the University of Tsukuba, Japan for establishing the culture of FKOI. TN is supported by a JSPS Research Fellowship for Young Scientists (DCI). This project was partially supported by grants from the National Science Foundation Microbial Genome Sequencing program (EF 08-27023) that was awarded to HSY, RAA, DB, from the Assembling the Tree of Life program (EF 04-3 I I I 7) that was awarded to $D B$, and a grant from the Korean Science and Engineering Foundation (MOST; ROI-2006-000-10207-0) that was awarded to SMB.

\section{References}

I. Bhattacharya D, Yoon HS, Hackett JD: Photosynthetic eukaryotes unite: endosymbiosis connects the dots. Bio Essays 2004, 26:50-60.

2. Palmer JD: The symbiotic birth and spread of plastids: how many times and whodunit? I Phycol 2003, 39:4- I2

3. Kalanon M, McFadden GI: The chloroplast protein translocation complexes of Chlamydomonas reinhardtii: a bioinformatic comparison of Toc and Tic components in plants, green algae and red algae. Genetics 2008, I79:95-II2.

4. Bhattacharya D, Medlin L: The phylogeny of plastids: A review based on comparisons of small-subunit ribosomal RNA coding regions. J Phycol 1995, 3 I:489-498.

5. Moreira D, Le Guyader H, Phillippe $H$ : The origin of red algae and the evolution of chloroplasts. Nature 2000, 405:69-72.

6. Yoon HS, Hackett JD, Pinto G, Bhattacharya D: The single, ancient origin of chromist plastids. Proc Natl Acad Sci USA 2002, 99: $15507-15512$

7. Yoon HS, Hackett JD, Van Dolah FM, Nosenko T, Lidie KL, Bhattacharya D: Tertiary endosymbiosis driven genome evolution in dinoflagellate algae. Mol Biol Evol 2005, 22: I 299-I308.

8. Tengs T, Dahlberg OJ, Shalchian-Tabrizi K, Klaveness D, Rudi K, Delwiche CF, Jakobsen KS: Phylogenetic analyses indicate that the 19'Hexanoyloxy-fucoxanthin- containing dinoflagellates have tertiary plastids of haptophyte origin. Mol Biol Evol 2000 , 17:718-729.

9. Cavalier-Smith T: Principles of protein and lipid targeting in secondary symbiogenesis: euglenoid, dinoflagellate, and sporozoan plastid origins and the eukaryote family tree. J Eukaryot Microbiol 1999, 46:347-366.

10. McFadden GI: Endosymbiosis and evolution of the plant cell. Curr Opin Plant Biol 1999, 2:513-519.

II. Yoon HS, Hackett JD, Ciniglia C, Pinto G, Bhattacharya D: A molecular timeline for the origin of photosynthetic eukaryotes. Mol Biol Evol 2004, 2 I:809-818.

12. Douzery EJ, Snell EA, Bapteste E, Delsuc F, Philippe H: The timing of eukaryotic evolution: does a relaxed molecular clock reconcile proteins and fossils? Proc Natl Acad Sci USA 2004, 101:15386-15391.

13. Bhattacharya D, Helmchen T, Melkonian M: Molecular evolutionary analyses of nuclear-encoded small subunit ribosomal RNA identify an independent rhizopod lineage containing the Euglyphina and the Chlorarachniophyta. J Eukaryot Microbiol 1995, 42:65-69.

14. Lauterborn R: Protozoenstudien II. Paulinella chromatophora nov. gen., nov. spec., ein beschalter Rhizopode des Sußwassers mit blaugrunen chromatophorenartigen Einschlussen. Z Wiss Zool 1895, 59:537-544.

15. Porter SM: The Proterozoic fossil record of heterotrophic eukaryotes. In Neoproterozoic geobiology and paleobiology Edited by: Xiao S, Kaufman AJ. The Netherlands: Springer; 2006:I-2I.

16. Porter SM, Knoll AH: Testate amoebae in the Neoproterozoic era: evidence from vase-shaped microfossils in the Chuar group, Grand Canyon. Paleobiol 2000, 26:360-385.

17. Marin B, Nowack EC, Melkonian M: A plastid in the making: evidence for a second primary endosymbiosis. Protist 2005, I 56:425-432.

18. Rodriguez-Ezpeleta N, Philippe H: Plastid origin: replaying the tape. Curr Biol 2006, I 6:R53-56. 
19. Kies L: Elektronenmikroskopische Untersuchungen an Paulinella chromatophora Lauterborn, einer Thekamobe mit blau-grunen Endosymbionten (Cyanellen). Protoplasma 1974, 80:69-89.

20. Melkonian M, Mollenhauer D: Robert Lauterborn (1869-1952) and his Paulinella chromatophora. Protist 2005, I 56:253-262.

21. Bhattacharya D, Archibald JM, Weber AP, Reyes-Prieto A: How do endosymbionts become organelles? Understanding early events in plastid evolution. Bio Essays 2007, 29: I239- 246.

22. Nowack EC, Melkonian M, Glockner G: Chromatophore genome sequence of Paulinella sheds light on acquisition of photosynthesis by eukaryotes. Curr Biol 2008, 18:410-418.

23. Reyes-Prieto A, Weber AP, Bhattacharya D: The origin and establishment of the plastid in algae and plants. Annu Rev Genet 2007, 41: |47-168.

24. Hannah F, Rogerson A, Anderson OR: A description of Paulinella indentata n. sp. (Filosea: Euglyphina) from subtidal coastal benthic sediments. J Eukaryot Microbiol 1996, 43: I-4.

25. Johnson PW, Hargraves PE, Sieburth JM: Ultrastructure and ecology of Calycomonas ovalis Wulff, (Chrysophyceae) and its redescription as a testate rhizopod, Paulinella ovalis n. comb. (Filosea: Euglyphina). J Protozool 1919, 35:618-626.

26. Vørs N: Marine heterotrophic amoebae, flagellates and Heliozoa from Belize (Central America) and Tenerife (Canary Islands), with descriptions of new species, Luffisphaera bulbochaete n. sp., L. longihastis n. sp., L. turriformis $\mathbf{n}$. sp. and Paulinella intermedia n. sp. J Eukaryot Microbiol 1993, 40:272-287.

27. Pendard E: Notes sur quelques sarcodines. Int Rev Suisse Zool 1905, 13:603-610.

28. Brown JM: On the occurrence of Paulinella chromatophora in Britain. Naturalist 19/5:157-I59.

29. Lackey JB: Some fresh water protozoa with blue chromatophores. Biol Bull 1936, 71:492-497.

30. Kepner WA: Paulinella chromatophora. Biol Bull 1905, 9:128-129.

31. Kies L, Kremer BP: Function of cyanelles in the Thecamoeba Paulinella chromatophora. Naturwissenschaften 1979, 66:578.

32. Marin B, Nowack EC, Glockner G, Melkonian M: The ancestor of the Paulinella chromatophora obtained a carboxysomal operon by horizontal gene transfer from a Nitrococcus-like gamma-proteobacterium. BMC Evol Biol 2007, 7:85.

33. Yoon HS, Reyes-Prieto A, Melkonian M, Bhattacharya D: Minimal plastid genome evolution in the Paulinella endosymbiont. Curr Biol 2006, 16:R670-672

34. Nikolaev SI, Berney C, Fahrni JF, Bolivar I, Polet S, Mylnikov AP, Aleshin VV, Petrov NB, Pawlowski J: The twilight of Heliozoa and rise of Rhizaria, an emerging supergroup of amoeboid eukaryotes. Proc Natl Acad Sci USA 2004, I 01:8066-807I.

35. Cavalier-Smith $\mathrm{T}$ : The phagotrophic origin of eukaryotes and phylogenetic classification of Protozoa. Int J Syst Evol Microbiol 2002, 52:297-354

36. Takishita K, Yubuki N, Kakizoe N, Inagaki Y, Maruyama T: Diversity of microbial eukaryotes in sediment at a deep-sea methane cold seep: surveys of ribosomal DNA libraries from raw sediment samples and two enrichment cultures. Extremophiles 2007, I I:563-576.

37. Tekle YI, Grant J, Cole JC, Nerad TA, Anderson OR, Patterson DJ, Katz LA: A multigene analysis of Corallomyxa tenera sp. nov. suggests its membership in a clade that includes Gromia, Haplosporidia and Foraminifera. Protist 2007, 158:457-472.

38. Martin W, Herrmann RG: Gene transfer from organelles to the nucleus: how much, what happens, and why? Plant Physiol 1998, I 1 8:9-17.

39. Moustafa A, Reyes-Prieto A, Bhattacharya D: Chlamydiae has contributed at least 55 genes to Plantae with predominantly plastid functions. PLoS ONE 2008, 3:e2205.

40. Nakayama T, Ishida K: Another acquisition of a primary photosynthetic organelle is underway in Paulinella chromatophora. Curr Biol 2009, 19:R284-285.

4I. Edgar RC: MUSCLE: a multiple sequence alignment method with reduced time and space complexity. BMC bioinformatics 2004, 5: II3.

42. Stamatakis A: RAxML-VI-HPC: maximum likelihood-based phylogenetic analyses with thousands of taxa and mixed models. Bioinformatics 2006, 22:2688-2690.
43. Guindon S, Gascuel O: A simple, fast, and accurate algorithm to estimate large phylogenies by maximum likelihood. Syst Biol 2003, 52:696-704.

44. Huelsenbeck JP, Ronquist F: MRBAYES: Bayesian inference of phylogenetic trees. Bioinformatics 200I, 17:754-755.

45. Yang Z: PAML 4: phylogenetic analysis by maximum likelihood. Mol Biol Evol 2007, 24:|586-159I.
Publish with Biomed Central and every scientist can read your work free of charge

"BioMed Central will be the most significant development for disseminating the results of biomedical research in our lifetime. "

Sir Paul Nurse, Cancer Research UK

Your research papers will be:

- available free of charge to the entire biomedical community

- peer reviewed and published immediately upon acceptance

- cited in PubMed and archived on PubMed Central

- yours - you keep the copyright 